\title{
Multi-periodic climate dynamics: spectral analysis of long-term instrumental and proxy temperature records
}

\author{
H.-J. Lüdecke ${ }^{1, *}$, A. Hempelmann ${ }^{2}$, and C. O. Weiss ${ }^{3, *}$ \\ ${ }^{1}$ HTW, University of Applied Sciences, Saarbrücken, Germany \\ ${ }^{2}$ University of Hamburg, Hamburg Observatory, Hamburg, Germany \\ ${ }^{3}$ Physikalisch-Technische Bundesanstalt, Braunschweig, Germany \\ * retired
}

Correspondence to: H.-J. Lüdecke (moluedecke@t-online.de)

Received: 16 August 2012 - Published in Clim. Past Discuss.: 12 September 2012

Revised: 20 January 2013 - Accepted: 4 February 2013 - Published: 22 February 2013

\begin{abstract}
The longest six instrumental temperature records of monthly means reach back maximally to $1757 \mathrm{AD}$ and were recorded in Europe. All six show a V-shape, with temperature drop in the 19th and rise in the 20th century. Proxy temperature time series of Antarctic ice cores show this same characteristic shape, indicating this pattern as a global phenomenon. We used the mean of the six instrumental records for analysis by discrete Fourier transform (DFT), wavelets, and the detrended fluctuation analysis (DFA). For comparison, a stalagmite record was also analyzed by DFT. The harmonic decomposition of the abovementioned mean shows only six significant frequencies above periods over $30 \mathrm{yr}$. The Pearson correlation between the mean, smoothed by a $15-\mathrm{yr}$ running average (boxcar) and the reconstruction using the six significant frequencies, yields $r=0.961$. This good agreement has a $>99.9 \%$ confidence level confirmed by Monte Carlo simulations. It shows that the climate dynamics is governed at present by periodic oscillations. We find indications that observed periodicities result from intrinsic dynamics.
\end{abstract}

\section{Introduction}

It is widely accepted that the mean surface temperature of the globe has been rising in the 20th century. However, how strong and how unusual this rise in comparison with the temperature variations over the last 250 or even $2000 \mathrm{yr}$ was is one of the subjects in the current climate debate. Long-range instrumental records going back to a maximum of about $250 \mathrm{yr}$ BP exist only in Central Europe.
But even here they are not abundant. The places with reliable monthly time series number six in all: Prague, Hohenpeißenberg, Kremsmünster, Vienna, Paris and Munich (CRU, 2012; DWD, 2012; Auer et al., 2007; Météo France, 2012; CHMI, 2012). They have some of the longest, most reliable instrumental temperature records as monthly means to be had anywhere. The time series of Paris begins in 1757 AD, whereas those of Hohenpeißenberg and Munich begin only in $1781 \mathrm{AD}$. Additionally, a high quality temperature proxy record from a stalagmite retrieved in the Spannagel Cave near Innsbruck (Austria) at $2347 \mathrm{~m}$ a.s.l. is available ranging from 90 BC until 1935 AD (Mangini et al., 2005). It has time steps between $1 \mathrm{yr}$ and $13 \mathrm{yr}$. Finally, a yearly $\delta^{18} \mathrm{O}$ record of the period 1801-1997 AD from the analysis of an Antarctic ice core is available for further comparison (Graf et al., 2002).

We used the temperature anomalies divided by the standard deviations for each of the above-cited six instrumental records from Central Europe. As these are rather close to one another, we used the mean of these (hereafter M6) for the numerical analysis. Equally the anomalies of the stalagmite record and the ice core record, divided by the standard deviations, were analyzed (hereafter SPA for the stalagmite record and IC for the ice core record).

Figure 1 depicts the six normalized time series whose average is M6. They all show a typical V-pattern with a maximum around the year $1800 \mathrm{AD}$, a fall until a minimum at about $1880 \mathrm{AD}$, and a rise until the recent maximum at about 2000 AD. Both maxima have similar magnitudes. Figure 2 gives M6 superimposed with IC showing the V-shape of the temperature history equally in the Northern and Southern 

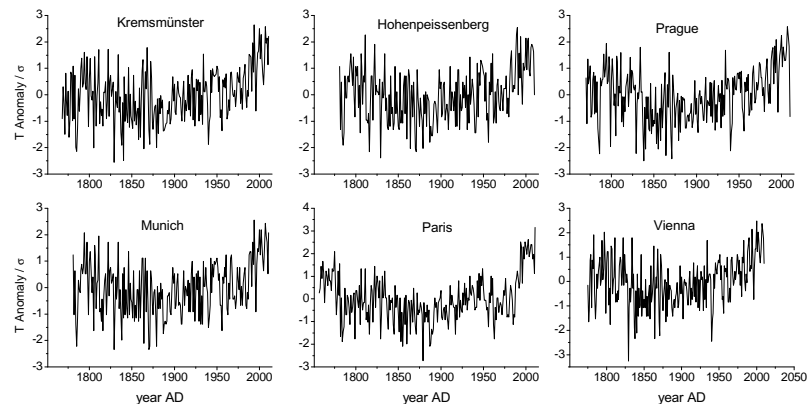

Fig. 1. Long-term temperature records from six central European stations.

Hemisphere, and therefore as a global phenomenon. We note that $\mathrm{NH}$ temperature reconstructions corroborate the temperature decrease in the 19th century (Crowley, 2000).

The time series M6 and SPA are analyzed by the discrete Fourier transform (DFT) with zero padding. Zero padding in the time domain increases the frequency steps and corresponds to an ideal interpolation in the frequency domain. To obtain information about the significance of the peaks, we adjoin in the DFT spectra the $90 \%$ and the $95 \%$ confidence limits of the background noise evaluated by Monte Carlo simulations using, for M6 and SPA each, 10000 surrogate records as a random background. Next, an empirical reconstruction of M6 based on the results of a DFT without zero padding is executed, which is restricted to oscillations of periods no shorter than $30 \mathrm{yr}$. The quality of the reconstruction suggests predicting near-future temperature changes as indicated in Fig. 6.

\section{The data basis}

The instrumental records from Central Europe consist of monthly means and are continuous except for Paris and $\mathrm{Mu}-$ nich, which are made up each of two parts from different nearby stations. However, both for Paris and Munich the major part of the two single time series covers nearly the whole record length. The monthly means are converted to yearly means for the application of the DFT. In contrast to this, both the stalagmite record SPA and the ice core record IC consist of yearly means. The details of the records used in this paper are as follows: Kremsmünster, monthly (1768-2010) (Auer et al., 2007); Hohenpeißenberg, monthly (1781-2010) (CRU, 2012); Prague, monthly (17702010) (CHMI, 2012); Paris-Le-Bourget, monthly (17571993) (Météo France, 2012); Paris-Montsouris, monthly (1994-2011) (Météo France, 2012); Munich-Riem, monthly (1781-2007) (Auer et al., 2007); Munich-Airport, monthly (2007-2011) (DWD, 2012); Vienna, monthly (1775-2010) (CRU, 2012); stalagmite record SPA, unequally yearly (-90-1935) (Mangini et al., 2005); and ice core record IC, yearly (1801-1997) (Graf et al., 2002).

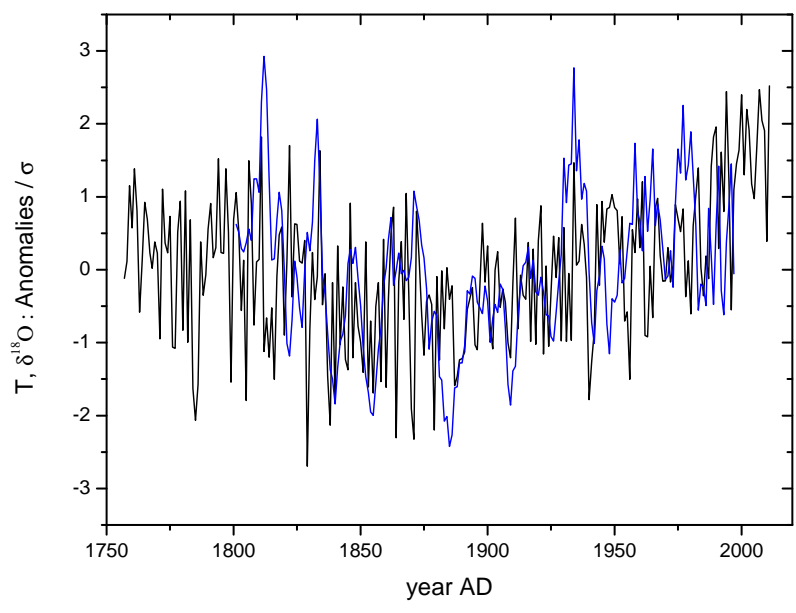

Fig. 2. Northern Hemisphere (Central European) instrumental temperature averaged record M6 (black) together with the Antarctic ice core record IC (blue) as a SH counterpart, each as an anomaly divided by the standard deviation.

\section{Methods}

In the literature, several methods for the spectral analysis of time series are reported (Ghil et al., 2002). We used in this study the simple method of the discrete Fourier transform (DFT). The time series M6 has equal time steps of one year, and, therefore, the DFT can be applied without any further complications. However, for SPA (which has unequal time steps) the DFT requires an interpolation procedure in the time domain. With the data $f_{k}$ in the time domain and $N$ as the number of $f_{k}$ (here $N=254$ ), the DFT is

$$
F_{j}=\frac{1}{N} \sum_{k=0}^{N-1} f_{k} W_{N}^{-k j}
$$

with $W_{N}=e^{2 \pi i / N}$ and the reverse transformation

$$
f_{k}=\sum_{j=0}^{N-1} F_{j} W_{N}^{k j} .
$$

In principle, for unequal time steps the DFT is not applicable. Interpolation in the time domain, however, can result in enhancing the low frequencies and reducing the high-frequency components (Schulz and Mudelsee, 2002). Therefore, we compared in a first step the result of the interpolated SPA record yielded by DFT with the result of the unmodified SPA record yielded by the Lomb-Scargle periodogram method. As an outcome, no significant differences in the peak frequencies but differences in the peak strengths occur between DFT with the interpolated SPA and the periodogram with the unchanged SPA. Because the stalagmite record SPA is predominantly used in this paper for a comparison of frequencies with M6 and because the widest time steps of SPA lie in the period from $90 \mathrm{BC}$ until $500 \mathrm{AD}$, we omitted the first 
years of $90 \mathrm{BC}$ until $500 \mathrm{AD}$ and carried out again the comparison of the DFT with the periodogram. As a result, we find for the shortened interpolated SPA, both for the frequencies and the power densities, good accordance of the DFT and the periodogram. To obtain more frequency steps, we generally applied zero padding in the DFT except for the empirical reconstruction of M6. Furthermore, the power values $F_{j}$ in Eq. (1) were normalized by dividing by the area comprised by the whole spectrum.

In order to obtain confidence levels of the background noise, the autocorrelation (persistence) of the records M6 and SPA has to be considered. The appropriate method for the evaluation of the persistence of a time series is the detrended fluctuation analysis (DFA) specified in Kantelhardt (2004), Lennartz and Bunde $(2009,2011)$ and references cited therein. In general, one visualizes the significance of peaks in DFT power spectra against the background noise with lines of $90 \%, 95 \%$ or $99 \%$ confidence levels and assumes a background of red noise as an AR1 process (Schulz and Mudelsee, 2002). We applied a more realistic procedure that accounts for the autocorrelation of the record: The autocorrelation (persistence) of a time series is characterized by its Hurst exponent $\alpha$, which can be evaluated with DFA. For the stalagmite record SPA, an $\alpha$ of 0.9 was already reported (Lüdecke, 2011), which indeed corresponds roughly to a red noise background. However, our DFA of M6 yielded a value of $\alpha=0.58 \pm 0.2$ (estimated). Because the DFA needs a minimum of 500 data points for the autocorrelation analysis of M6, the monthly mode of M6 had to be applied. To eliminate seasonal influences, we subtracted the seasonal mean value from the data and divided by the seasonal standard deviation. This yields a normalized record without seasonal effects that is appropriate for the DFA (Lennartz and Bunde, 2009). The result of $\alpha=0.58$ corresponds to other values for temperature series (Rybski and Bunde, 2009) and demonstrates that red noise as a background for M6 is not adequate. Therefore, one has to ascertain that the random records of the Monte Carlo method simulating the background noise have the appropriate $\alpha$ values. For this purpose we generated surrogate records with the Hurst exponents $\alpha=0.9$ for SPA and $\alpha=0.58$ for M6 by using a standard method (Turcotte, 1997).

The DFT without zero padding was applied for the reconstruction of M6 (Eqs. 1 and 2). Here, we chose the basic method of selecting specific frequencies for the reverse transformation (Eq. 2) resulting directly in the reconstruction without any further optimization procedures. Choosing among the lowest eight frequencies the ones with periods no shorter than $30 \mathrm{yr}$ (definition of "climate" as 30-yr temperature average), the six with the highest power densities yield an excellent reconstruction of M6. No other spectral contributions were used.

\section{DFT and wavelet analysis}

The results of the DFT are shown in Fig. 3. The left panel of Fig. 3 depicts the power densities of the DFT for M6 with padded zeros together with the $90 \%$ and $95 \%$ curves of confidence yielded by the Monte Carlo simulations. The right panel shows the same for SPA. Figure 5 shows the wavelet spectrum for the interpolated stalagmite record SPA. For M6 a wavelet analysis is meaningless because of the shortness of the time series. Four of our six selected frequencies in M6 have a confidence level over $95 \%$ and only one over $99 \%$. We find for SPA roughly the periods corresponding to 250 , 80,65 , and $35 \mathrm{yr}$ from M6. A conspicuous disagreement is found for the peak with the period of $100 \mathrm{yr}$, which is very strong in SPA and nearly lacks in M6.

We note that the $\sim 250$-yr peak in Fig. 3 left results from only about one $250-y r$ period, which covers the entire record length. This is clearly insufficient to detect real oscillatory dynamics. The question thus, if there is in fact a 250 -yr periodicity, can only be decided by longer records. Fortunately the SPA record covers about 2000 yr. Its spectrum shows a strong peak at $\sim 235 \mathrm{yr}$ (Fig. 3 right). Additionally, Fig. 4 shows that the 235-yr component of SPA follows roughly the overall shape of SM6. We have also ascertained that the appearance of the $\sim 250$-yr period is not an artifact of the 254 -yr record length of M6 by extending M6 by $100 \mathrm{yr}$ with white noise random numbers. Also this 354 -yr-long extended record yields the prominent $\sim 250$-yr period. This and Fig. 4 suggest that the $\sim 250$-yr peak in Fig. 3 left is not an artifact and corresponds to a real oscillation. The wavelet diagram shows that this cycle has been the dominant one since about $1100 \mathrm{AD}$ (see Fig. 5) corresponding to the dominant strength of this cycle in M6.

The wavelet analysis (Fig. 5) shows that historically a $\sim 125$-yr cycle was dominating the Earth temperature. During the many decades this cycle has weakened and the strength shifted to the subharmonic (see Sect. 7) of $\sim 250 \mathrm{yr}$, which is now the dominant periodicity. In addition, the four lowest frequencies in Fig. 3 (right) show rather precisely the spectral pattern of the generation of the subharmonics $(1,0.5$, $0.75,1.25$, which correspond to the evaluated periods 234 , 512, 341, 182 yr of Fig. 3 right). Such subharmonic generation is characteristic of the transition from periodic to chaotic oscillations of dynamic systems (Feigenbaum, 1978, 1983).

\section{Empirical reconstruction of the mean record M6}

Our empirical reconstruction of M6 (hereafter RM6) with $N=254$ data points is based on the DFT without zero padding (Eq. 1). For the inverse transformation (Eq. 2) we selected six periods (frequencies $>0$ ) obeying the conditions that they are longer than $30 \mathrm{yr}$ and yield the strongest power densities among the first 8 DFT-frequencies $i / N, i=$ $\{1, \ldots, 8\}$. As a result, the reconstruction is 

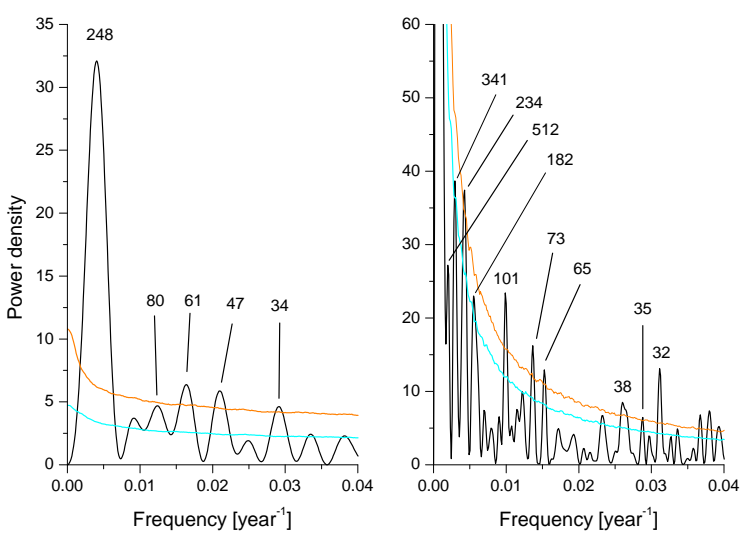

Fig. 3. Left panel: DFT of M6 (average from six central European instrumental time series). Right panel: same for SPA, interpolated time series of a stalagmite from the Austrian Alps for the period 500-1935 AD. In both DFT analyses the records were padded with zeros. The upper confidence curve (brown) is for $95 \%$, the lower (cyan) for $90 \%$ against background noise, each of those established by 10000 Monte Carlo runs. The most relevant peaks are indicated by their period length.

$f_{k}=\sum_{j} F_{j} W_{N}^{k j} ; \quad j=0,1,3,4,5,6,7$

$\operatorname{RM} 6(t)=\sum_{j} a_{j} \cos (2 \pi j t / N)+b_{j} \sin (2 \pi j t / N) ;$

$j=0,1,3,4,5,6,7$

The parameters of Eq. (4) are given in Table 1.

Figure 6 depicts the comparison of the reconstruction RM6 and the record M6 after being boxcar-smoothed over $15 \mathrm{yr}$ (hereafter SM6).

\section{Confidence level of the reconstruction}

The Pearson correlation of the smoothed record SM6 with the reconstruction RM6 (black and red curves in Fig. 6) has a value of $r=0.961$. In order to ascertain the statistical confidence level of this accordance, we assumed a null hypothesis and evaluated it by Monte Carlo simulations based on random surrogate records of the same length and the same Hurst exponent $(\alpha=0.58)$ as M6 generated by a standard method (Turcotte, 1997) (the surrogate records hereafter SU, and the boxcar-smoothed SU over $15 \mathrm{yr}$ hereafter SSU). As the null hypothesis we assumed that the accordance of the reconstruction RM6 with SM6 is caused by chance. We applied 10000 surrogate records SU. Each of the record was analyzed following the same procedure as for M6. Next, for each surrogate SU the reconstruction was generated that used - again following the procedure as for M6 - six frequencies

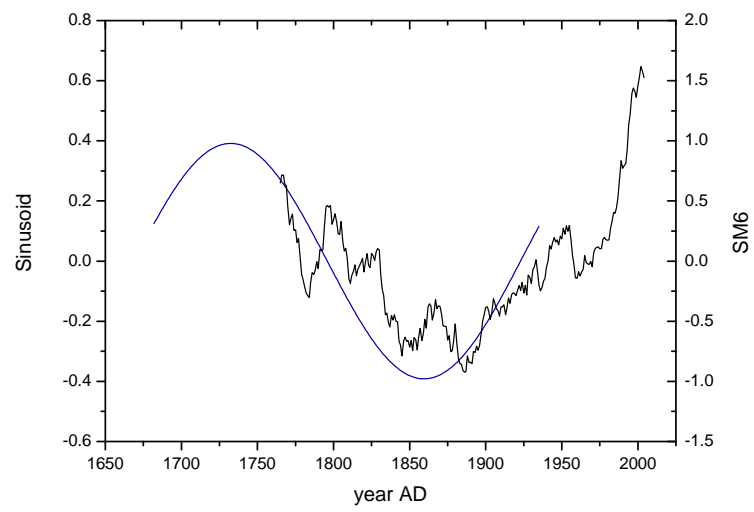

Fig. 4. Temporal picture of the $\sim 235 \mathrm{yr}$ frequency component of SPA (sinusoid, blue) together with SM6 (black), showing an approximate matching of the phases.

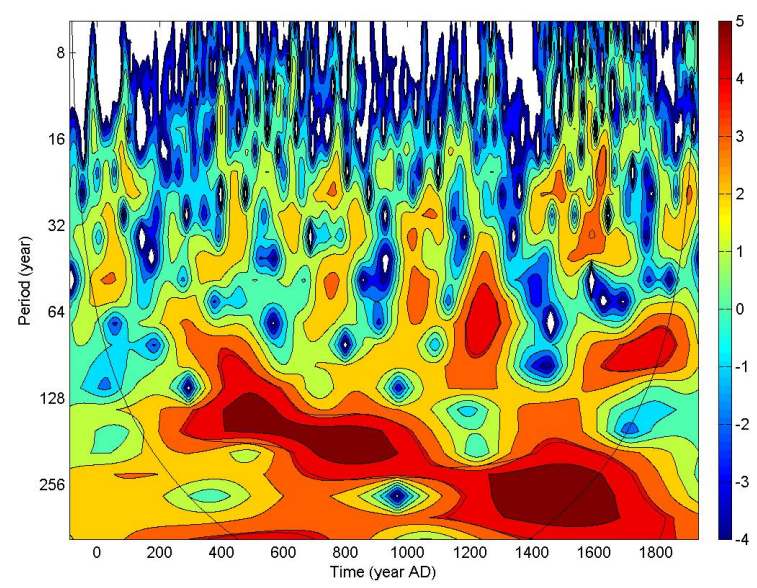

Fig. 5. Wavelet (Morlet) spectrum (Torrence and Compo, 1998) of the interpolated SPA record for the period -90 until 1935 AD. The solid black line from the left to the right top of the figure is the cone of influence (below the cone of influence, the results are not significant). The spectrum shows that the power density transfers with time from the $\sim 125$-yr cycle to the $\sim 250$-yr cycle (period doubling).

with the strongest power densities among the first eight frequencies of the DFT without zero padding. Finally, the Pearson correlation of this reconstruction with SSU was evaluated. As a result, among $10000 \mathrm{SU}$ we found one surrogate record with the maximal $r=0.960,9$ records with $r \geq 0.95$, and 53 records with $r \geq 0.94$. Therefore, the null hypothesis could be rejected with a confidence level of $>99.9 \%$.

\section{Discussion}

The temperature records from six Central European stations show remarkable agreement, justifying their averaging to produce a "Central European temperature record" M6. The characteristic of these records, namely the pronounced 
Table 1. Frequencies, periods, and the coefficients $a_{j}$ and $b_{j}$ of the reconstruction RM6 due to Eqs. (1), (3), and (4) $(N=254)$.

\begin{tabular}{rrrrr}
\hline$j$ & $j / N\left(\mathrm{yr}^{-1}\right)$ & Period $(\mathrm{yr})$ & $a_{j}$ & $b_{j}$ \\
\hline 0 & 0 & - & 0 & 0 \\
1 & 0.00394 & 254 & 0.68598 & -0.12989 \\
2 & 0.00787 & 127 & - & - \\
3 & 0.01181 & 85 & 0.19492 & -0.14677 \\
4 & 0.01575 & 64 & 0.17465 & -0.22377 \\
5 & 0.01968 & 51 & 0.14730 & -0.10810 \\
6 & 0.02362 & 42 & -0.02510 & -0.12095 \\
7 & 0.02756 & 36 & 0.12691 & 0.01276 \\
8 & 0.03150 & 32 & - & - \\
\hline
\end{tabular}

minimum around 1880, is equally found in Antarctic ice core temperature data, which are also overall in agreement with M6, revealing this 1880 minimum as a global phenomenon.

The Fourier transform of M6 yields pronounced spectral peaks, indicating dynamics by periodicity. Indeed, the reconstruction, in which nothing but the six strongest frequency components (with periods $>30 \mathrm{yr}$ ) are retained, yields excellent agreement with the measurement M6. A temperature record obtained from a Central European stalagmite is also Fourier-analyzed and shows, for comparison, similar periodicities. It confirms the 250 -yr period yielded by the DFT of M6 as a real and dominant oscillation. The cause of the periodicities is not known to us. We would think that they constitute intrinsic system dynamics as it is generally found for dissipative systems with energy input (here the Earth dissipating the radiative energy provided by the Sun). Such dynamics are the physics behind all terrestrial weather dynamics (e.g., trade winds or El Niño). This interpretation as intrinsic system dynamics is supported by the wavelet analysis of the stalagmite data. The latter shows a drift over $1600 \mathrm{yr}$ of peak intensity from the 128-yr period to the 256-yr period. A further doubling to $\sim 500 \mathrm{yr}$ (peak visible in the spectrum, right Fig. 3) causes the recent weakening of the 250-yr period, visible in the wavelet diagram. Such a shifting of energy from a fundamental to a subharmonic frequency component is characteristic of the Feigenbaum universal scenario of transition to chaos by a cascade of subharmonics, for nonlinear, dissipative systems with energy input (Feigenbaum, 1978, 1983). Whereas harmonic generation is just an expression of nonlinearity, subharmonic generation is peculiar to the Feigenbaum scenario, since it requires, different from harmonic generation, a particular phase-matching mechanism for sum- and difference-frequencies generated by the system non-linearities. The four lowest frequency lines of Fig. 3 (right) give additionally evidence of the climate oscillations as intrinsic dynamics. They show the pattern characteristic of generation of two subharmonics, which corresponds rather convincingly to the Feigenbaum transition to chaos.

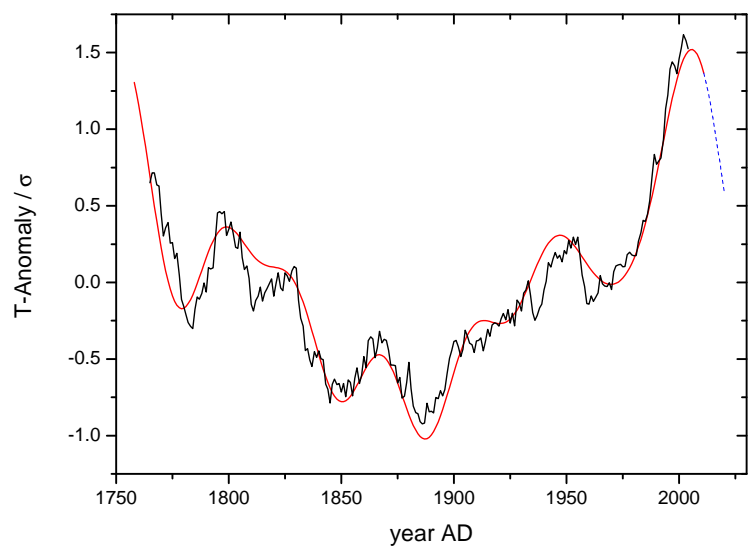

Fig. 6. (color online) $15 \mathrm{yr}$ running average record SM6 (black); reconstruction RM6 according to Eqs. (1), (3) and (4) (red); projection of future $\mathrm{NH}$ temperatures mainly due to the $\sim 65$-yr periodicity (dashed blue)

The agreement of the reconstruction of the temperature history using only the six strongest components of the spectrum, with M6, shows that the present climate dynamics is dominated by periodic processes. This does not rule out a warming by anthropogenic influences such as an increase of atmospheric $\mathrm{CO}_{2}$. Such secular effects could have been incorporated by the DFT, e.g., into the $250-\mathrm{yr}$ cycle obtained from M6, and would then not show up as a discrepancy between SM6 and RM6.

The prediction of a temperature drop in the near future results essentially from the $\sim 64$-yr cycle, which to our knowledge is the Atlantic (Pacific) Multidecadal Oscillation (Mantua and Hare, 2002; Hurrel and van Loon, 1997). Four periods of the 64-yr cycle are clearly visible in Fig. 6, and the Atlantic (Pacific) Multidecadal Oscillation can be traced back for more than $1000 \mathrm{yr}$. This cycle was identified by Schlesinger and Ramankutty (1994) as intrinsic dynamics. Tsonis et al. (2007) found a mechanism in observed climate indices of the period 1900-2005, which is consistent with the theory of synchronized chaos. On the other hand external causes for periodic dynamics exist also, such as planetary motion, solar cycles and atmosphere-ocean-sea-ice interaction (Scafetta, 2012a,b,c; Solheim et al., 2012; Dima and Lohmann, 2007).

While thus many indications point to the oscillations as intrinsic dynamics of the Earth, external causes for periodic dynamics cannot be ruled out. Strictly speaking, even the observed periodic-doubling could be that of an external forcing with just this dynamics. 


\section{Appendix A}

\section{Abbreviations of the time series in this paper}

- IC: ice core record as yearly $\delta^{18} \mathrm{O}$ values.

- M6: mean of six instrumental records in Central Europe (both monthly and yearly).

- RM6: reconstruction of M6.

- SPA: stalagmite time series as yearly temperatures yielded by interpolation.

- SM6: M6 smoothed over 15 yr (boxcar).

- SU: surrogate records with Hurst exponents $\alpha=0.58 \pm$ 0.3 .

- SSU: smoothed SU over 15 yr (boxcar).

Acknowledgements. We thank Régine Larrieu (Météo france, Toulouse) for kindly allocating the Paris time series for our studies. We thank Luboš Motl for his technical information about the Prague record. We acknowledge helpful suggestions by the participants of the review process.

Edited by: E. Zorita

\section{References}

Auer, I., Böhm, R., Jurkovic, A., Lipa, W., Orlik, A., Potzmann, R., Schöner, W., Ungersböck, M., Matulla, C., Briffa, K., Jones, P., Efthymiadis, D., Brunetti, M., Nanni, T., Maugeri, M., Mercalli, L., Mestre, O., Moisselin, J.-M., Begert, M., MüllerWestermeier, G., Kveton, V., Bochnicek, O., Stastny, P., Lapin, M., Szalai, S., Szentimrey, T., Cegnar, T., Dolinar, M., GajikCapka, M., Zaninovic, K., Majstorovic, Z., and Nieplova, E.: HISTALP - historical instrumental climatological surface time series of the Greater Alpine Region, Int. J. Climatol., 27, 17-46, 2007.

Climatic Research Unit (CRU): University of East Anglia (UK), available at: www.cru.uea.ac.uk/cru/data/temperature/ station-data/ (last access: August 2012), 2012.

Crowley, T. J.: Causes of Climate Change Over thre past 1000 Years, Science, 289, 270-277, 2000.

Czech Hydrometeorological Institute (CHMI): 14306 Praha 4 Czech Republic, available at: http://zmeny-klima.ic.cz/ klementinum-data/ (last access: August 2012), 2012.

Deutscher Wetterdienst (DWD): Frankfurter Straße 135, 63067 Offenbach (Germany), available at: www.dwd.de (last access: August 2012), 2012.

Dima, M. and Lohmann, G.: A Hemispheric Mechanism for the Atlantic Multidecadal Oscillation, J. Climate, 20, 2706-2719, 2007.

Feigenbaum, M. J.: Quantitative universality for a class of nonlinear transformations, J. Stat. Phys., 19, 25-51, 1978.

Feigenbaum, M. J.: Universal behavior in nonlinear systems, Physica D, 7, 16-39, 1983.
Ghil, L., Allen, M. R., Dettinger, M. D., Ide, K., Kondrashov, D., Mann, M. E., Robertson, A. W., Saunders, A., Tian, Y., Varadi, F., and Yiou, P.: Advanced spectral methods for climatic time series, Rev. Geophys., 40, 1-41, 2002.

Graf, W., Oerter, H., Reinwarth, O., Stichler, W., Wilhelms, F., Miller, H., and Mulvaney, R.: Stable-isotope records from Dronning Maud Land, Antarctica, Ann. Glaciol., 35, 195-201, 2002.

Hurrel, J. W. and van Loon, H.: Decadal Variations in Climate Associated with the North Atlantic Oscillation, Climatic Change, 36, 301-326, 1997.

Kantelhardt, J. W.: Fluktuationen in komplexen Systemen, Habilitationsschrift, Universität Gießen, Germany, 19 June, available at: www.physik.uni-halle.de/Fachgruppen/kantel/habil.pdf (last access: August 2012), 2004.

Lennartz, S. and Bunde, A.: Trend evaluation in records with longterm memory: application to global warming, Geophys. Res. Lett., 36, L16706, doi:10.1029/2009GL039516, 2009.

Lennartz, S. and Bunde, A.: Distribution of natural trends in longterm correlated records: a scaling approach, Phys. Rev., 84, 021129, doi:10.1103/PhysRevE.84.021129, 2011.

Lüdecke, H.-J.: Long-term instrumental and reconstructed temperature records contradict anthropogenic global warming, Energy \& Environment, 22, 723-745, 2011.

Mangini, A., Spötl, C., and Verdes, P.: Reconstruction of temperature in the Central Alps during the past 2000 years from a ${ }^{18} \mathrm{O}$ stalagmite record, Earth Planet. Sc. Lett., 235, 741-751, 2005.

Mantua, N. J. and Hare, S. R.: The Pacific Decadal Oscillation, J. Oceanogr., 58, 35-44, 2002.

Météo France: available at: http://france.meteofrance.com (last access: 21 February 2013), 42 Av. Gaspard Coriolis, 31057 Toulouse Cedex (France), 2012.

Rybski, D. and Bunde, A.: On the detection of trends in long-term correlated records, Physica A, 388, 1687-1695, 2009.

Scafetta, N.: Testing an astronomically based decadal-scale empirical harmonic climate model versus the general circulation climate models, J. Atmos. Sol.-Terr. Phy., 80, 124-137, 2012a.

Scafetta, N.: Multi-scale harmonic model for solar and climate cyclical variation throughout the Holocene based on JupiterSaturn tidal frequencies plus the 11-yr solar dynamo cycle, J. Atmos. Sol.-Terr. Phy., 80, 296-311, 2012b.

Scafetta, N.: Does the Sun work as a nuclear fusion amplifier of planetary tidal forcing? A proposal for a physical mechanism based on the mass-luminosity relation, J. Atmos. Sol. Terr.-Phy., 81-82, 27-40, 2012c.

Schlesinger, E. and Ramankutty, N.: An oscillation in the global climate system of period 65-70 years, Nature, 367, 723-726, 1994.

Schulz, M. and Mudelsee, M.: REDFIT: estimating red-noise spectra directly from unevenly spaced paleoclimatic time series, Comput. Geosci., 28, 421-426, 2002.

Solheim, J.-E., Stordahl, K., and Humlum, O.: The long sunspot cycle 23 predicts a significant temperature decrease in cycle 24 , J. Atmos. Sol.-Terr. Phy., 80, 267-284, 2012.

Torrence, C. and Compo, P.: A practical guide to wavelet analysis, B. Am. Meteorol. Soc., 79, 61-78, 1998.

Tsonis, A. A., Swanson, K., and Kravtsov, S.: A new dynamical mechanism for major climate shifts, Geophys. Res. Lett., 34, L13705, doi:10.1029/2007GL030288, 2007.

Turcotte, D. L.: Fractals and Chaos in Geology and Geophysics, 2nd Edn., Cambridge University Press, Cambridge, 1997. 